\title{
Progression of nuclear sclerosis based on changes in refractive values after lens-sparing vitrectomy in proliferative diabetic retinopathy
}

This article was published in the following Dove Press journal:

Clinical Ophthalmology

I6 April 2014

Number of times this article has been viewed

\section{Tsunehiko Ikeda' \\ Masahiro Minami' \\ Kimitoshi Nakamura ${ }^{2}$ \\ Teruyo Kida' \\ Masanori Fukumoto' \\ Takaki Sato' \\ Eisuke Ishizaki'}

'Department of Ophthalmology, Osaka Medical College, Osaka, Japan; ${ }^{2}$ Nakamura Eye Clinic, Nagano, Japan
Correspondence:Tsunehiko Ikeda Department of Ophthalmology, Osaka Medical College, 2-7 Daigaku-cho, Takatsuki City, Osaka 569-8686, Japan Tel $+8 \mid 726846434$

Fax +8I 726820995

Email tikeda@poh.osaka-med.ac.jp
Background: Nuclear sclerosis (NS) based on the Emery-Little classification and refractive values after lens-sparing vitrectomy was compared between proliferative diabetic retinopathy (DR) patients and nondiabetic patients.

Methods: Progression of NS based on the Emery-Little classification and changes in refractive values were compared between 13 proliferative DR patients (14 eyes, DR group) and 14 nondiabetic patients (14 eyes, non-DR group) who underwent lens-sparing vitrectomy. All patients revealed grade I NS based on the Emery-Little classification. Mean patient age and refractive value just after surgery were 56.07 years and -0.33 diopters (D) in the DR group, and 57.06 years and $-0.96 \mathrm{D}$ in the non-DR group.

Results: The Emery-Little classification in the DR group at 6 and 24 months postoperative were grade I (13 eyes)/grade II (one eye) and grade I (eleven eyes)/grade II (three eyes), respectively. Mean refractive values in the DR group at 6, 12, and 24 months postoperative were $+0.28 \mathrm{D},+0.27 \mathrm{D}$, and $+0.37 \mathrm{D}$, respectively. The Emery-Little classification in the non-DR group at 6 and 24 months (or preoperative for patients undergoing cataract surgery) were grade I (five eyes)/grade II (eight eyes) and grade I (zero eyes)/grade II (eight eyes)/grade III (five eyes), respectively. The mean refractive value in the non-DR group at 6 months postoperative was $-3.20 \mathrm{D}$. All eyes exhibited myopic changes and progression of NS.

Conclusion: The findings of this study show that the progression of NS postvitrectomy is mild, even for DR patients 50 years of age or older, thus suggesting the need to reconsider the indications for simultaneous cataract surgery with vitrectomy.

Keywords: Emery-Little classification

\section{Introduction}

Vitreous surgery is used to treat many types of vitreoretinal disease, and it is often performed in phakic eyes as well as aphakic and pseudophakic eyes. It is reported that lens-sparing vitrectomy in patients over 50 years of age is associated with postoperative nuclear sclerosis (NS) and the development of myopia. ${ }^{1-5}$ The occurrence of progressive NS postvitrectomy was first described in patients undergoing vitrectomy for epiretinal membrane causing macular pucker. ${ }^{1}$ Numerous reports have shown that as many as $98 \%$ of older individuals who undergo vitreous surgery will develop clinically significant NS within 2 years postoperative. ${ }^{1}$ However, it has been reported that the progression of nuclear cataracts following vitrectomy for proliferative diabetic retinopathy (PDR) patients is slower than that in nondiabetic patients. ${ }^{6}$ To date, there has been no report which compared postoperative refractive values between these two groups. The purpose of the present retrospective study 
was to investigate and compare the progression of NS based on both the Emery-Little classification and postoperative refractive values after lens-sparing vitrectomy between patients 50 years of age or older with PDR and similar-age nondiabetic patients.

\section{Materials and methods}

This study involved 13 patients (14 eyes) 50 years of age or older who underwent lens-sparing vitrectomy for PDR and were followed-up for $\geq 2$ years postoperatively without complications (DR group), and a control group comprised of 14 nondiabetic patients (14 eyes) 50 years of age or older who underwent lens-sparing vitrectomy for other disorders (non-DR group). In both groups, the criteria for performing lens-sparing vitrectomy was that the patients have either no cataracts or only mild cataracts (grade I of the Emery-Little classification in all cases) which had little influence on visual acuity.

In the DR group, patient age ranged from 50 to 65 years (mean age: $56.07 \pm 5.77$ years), and the condition leading to surgery was vitreous hemorrhage in eight eyes, macular traction retinal detachment in three eyes, and extramacular traction retinal detachment in three eyes. Fluid-gas (20\% sulfur hexafluoride) exchange during surgery was performed in five eyes.

In the non-DR group, patient age ranged from 53 to 68 years (mean age: $57.06 \pm 5.05$ years), similar to that in the DR group, and the primary disease being treated was branch retinal vein occlusion in four eyes, central retinal vein occlusion in two eyes, macular hole in four eyes (stage III in three eyes and stage IV in one eye), macular epiretinal membrane in three eyes, and age-related macular degeneration in one eye. All cases of branch retinal vein occlusion, central retinal vein occlusion, and age-related macular degeneration were complicated with vitreous hemorrhage. Fluid-gas (20\% sulfur hexafluoride) exchange during surgery was performed in four eyes with macular hole.

Postoperative progression of NS was evaluated based on the Emery-Little classification and changes in refractive values. NS based on the Emery-Little grade and baseline refractive values just after surgery (in eyes with fluid-gas exchange: when the intraocular gas disappeared) and those at 6,12 , and 24 months postoperative were compared. The refractive values in all cases were measured by use of an autorefract/keratometer (ARK-1 ${ }^{\circledR}$; NIDEK Co., Ltd., Gamagori, Japan). In the DR group, refractive values in each patient's fellow eye were also compared during the 24-months follow-up period.

\section{Results}

Based on the Emery-Little classification, 13 eyes remained grade I at 6 months and eleven eyes remained grade I, and only three eyes progressed from grade I to grade II at 24 months postoperative in the DR group. On the other hand, eight eyes progressed from grade I to grade II at 6 months, and all eyes progressed from grade I to greater than grade II (five eyes from grade I to grade III) at 24 months postoperative (or preoperative for eyes that underwent cataract surgery) in the non-DR group.

Mean refractive values in the DR group at 6,12 , and 24 months postoperative were +0.28 diopters $(\mathrm{D}),+0.27 \mathrm{D}$, and $+0.37 \mathrm{D}$, respectively. Slight hyperopic changes tended to be seen, with almost no NS (Table 1). The mean refractive value in the non-DR group at 6 months postoperative was $-3.20 \mathrm{D}$. The mean duration of intraocular gas tamponade was $14 \pm 0.9$ days.

Changes in the Emery-Little grade and refractive values in the DR group showed no progression of NS in most of the operated eyes even after 24 months postoperative, yet the development of slight hyperopia was observed. In addition, no development of NS and myopic change were seen in the fellow eyes (Figure 1A). None of the DR-group eyes required cataract surgery during the follow-up period. In six patients (seven eyes) who were followed-up for $\geq 6$ years postoperative, no significant myopic changes (Figure 1B) or progression of NS was observed (Figure 2A-C).

Changes in refractive values in the non-DR group revealed significant myopic changes at 6 months postoperative, and eight eyes underwent cataract surgery due to the progression of NS from 6 to 12 months postoperative (Table 2). In addition, another five eyes underwent cataract surgery from 12 to 24 months postoperative. In all 13 eyes, the mean postoperative period until cataract surgery was $10.1 \pm 5.2$ months. The corrected visual acuity of the patients who underwent cataract surgery ranged from $20 / 200$ to $20 / 40$. In the DR group, the mean refractive values of the cases with and without fluid-gas exchange at 24-months postoperative were $+0.39 \mathrm{D}$ and $+0.36 \mathrm{D}$, respectively. In the non-DR group, the mean refractive values of the cases with and without fluid-gas exchange at 6 months

Table I Mean refractive values in the diabetic retinopathy group

\begin{tabular}{lll}
\hline & Operated eye & Fellow eye \\
\hline Just after surgery & $-0.33 \pm 1.90 D^{*}$ & $+0.27 \pm 1.79 \mathrm{D}$ \\
After 24 months & $+0.12 \pm 2.04 \mathrm{D}^{*}$ & $+0.21 \pm 1.90 \mathrm{D}$ \\
\hline
\end{tabular}

Notes: No myopic change was observed both in the operated eyes and the fellow eyes; *Represents $P=0.101$ (no significant difference in Student's $t$-test). Abbreviation: D, diopters. 
A

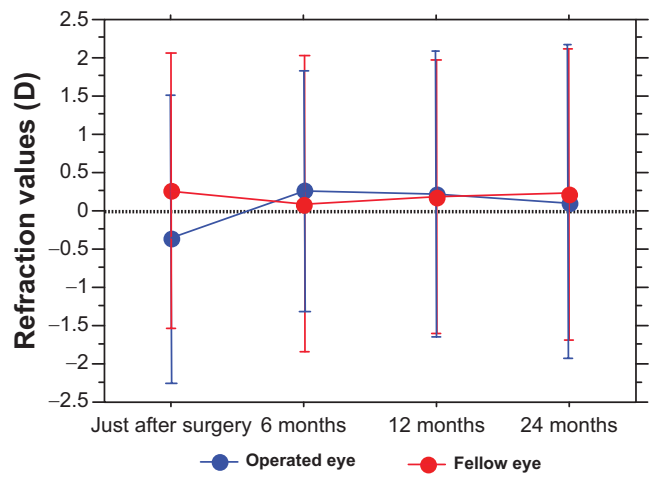

B

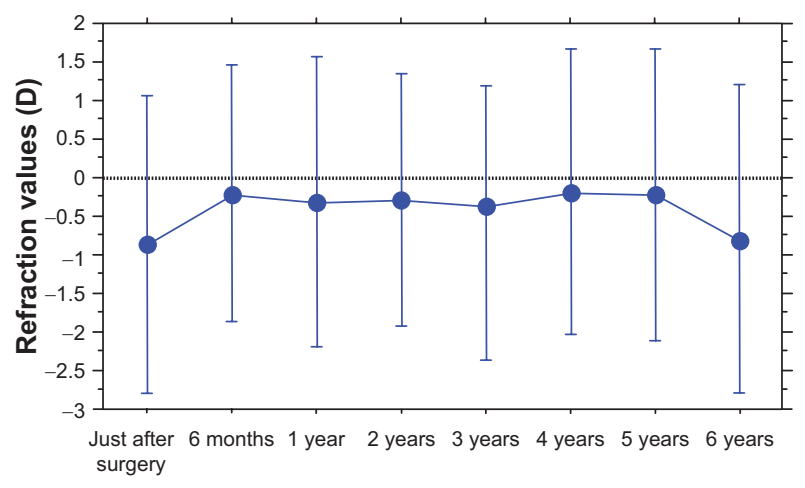

Figure I Changes in refractive values.

Notes: No development of myopia was found in the operated eye (A), even after 24 months postoperative in the diabetic retinopathy (DR)-group patients. Changes in refractive values in six eyes of the DR group (B). No development of myopia was found in the operated eye, even after 6 years postoperative.

Abbreviation: D, diopters.

postoperative were $-3.24 \mathrm{D}$ and $-3.18 \mathrm{D}$, respectively. There were no differences in refractive values between the cases with and without fluid-gas exchange during surgery.

\section{Discussion}

It has also been reported that with the progression of NS of the lens associated with aging, refractive values become more myopic. ${ }^{7}$ In the present study, the evaluation of the progression of NS was based on the Emery-Little classification and the changes in refractive values. Our findings revealed that in the DR group, there was no progression of NS during the 24-months follow-up period following vitrectomy. However, significant progression of NS was observed in the non-DR group.

A higher rate of cortical and posterior subcapsular opacification and probability of cataract surgery has been reported in diabetic patients compared to nondiabetic patients. ${ }^{8}{ }^{9}$ However, in those studies, diabetes mellitus was reportedly not associated with an increased risk of nuclear sclerotic cataract. Fluid-gas exchange reportedly increases the rate of progression of NS postvitrectomy, ${ }^{10}$ yet no
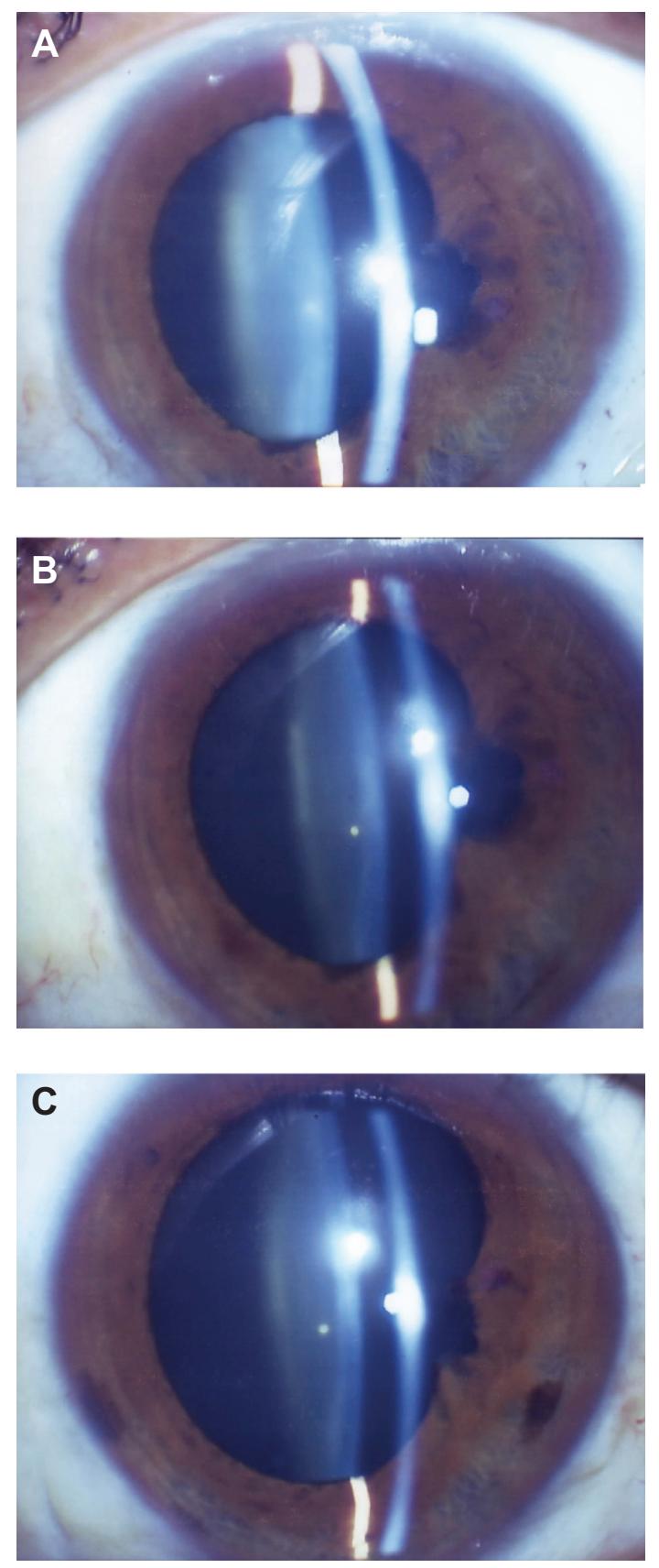

Figure 2 Change of nuclear sclerosis (NS) in a typical case of the diabetic retinopathy group.

Notes: In this case, no progression of NS occurred, even after 24 months postoperative. (A) Just after surgery (refractive values $=-0.25 \mathrm{D}$, Emery-Little grade I, visual acuity [VA]: 0.5 [0.7]). (B) After 12 months postoperative (refractive values $=+0.25 \mathrm{D}$, Emery-Little grade I, VA: 0.4 [0.6]). (C) After 24 months postoperative (refractive values $=+0.25 \mathrm{D}$, Emery-Little grade I, VA: 0.4 [0.6]). Abbreviation: D, diopters.

progression of NS was observed in the five eyes in the DR group in the present study that underwent fluid-gas exchange. However, the operated DR-group eyes did develop slight postoperative hyperopia. Although the cause is unclear, changes in anterior chamber depth following vitrectomy might be one reason in phakic eyes. ${ }^{11}$ 
Table 2 Mean refractive values in the nondiabetic retinopathy group

\begin{tabular}{lll}
\hline & Operated eye & Fellow eye \\
\hline Just after surgery & $-0.96 \pm 2.03 \mathrm{D}^{*}$ & $-0.86 \pm \mathrm{I} .64 \mathrm{D}$ \\
After 6 months & $-3.20 \pm 2.08 \mathrm{D} *$ & $-0.90 \pm \mathrm{I} .7 \mathrm{I} \mathrm{D}$ \\
After 12 months & $-4.4 \mathrm{I} \pm \mathrm{I} .99 \mathrm{D}$ (6 eyes) & $-1.07 \pm 2.0 \mathrm{I} \mathrm{D}$ (6 eyes) \\
After 24 months & $-2.25 \mathrm{D} \rightarrow-5.0 \mathrm{D}$ (I eye) & $-2.50 \mathrm{D}$
\end{tabular}

Notes: Myopic change was observed in the operated eyes. Cataract surgery was required in eight eyes by 12 months postoperative and in another five eyes by 24 months postoperative; *Represents $P<0.000$ I (significant difference in Student's $t$-test). Abbreviation: $\mathrm{D}$, diopters.

A previous study investigating the crystalline lens following vitrectomy for DR reported that moderate or greater progression of NS occurred in $7 \%$ of the cases. ${ }^{12}$ Furthermore, it has occasionally been reported that less postvitrectomy NS occurs in DR patients compared to nondiabetic patients. ${ }^{13,14}$ It has also been reported that at the 2-year follow-up point following lens-sparing vitrectomy in a DR group compared to a non-DR group, a significantly lower rate of cataract extraction occurred in the DR group $(P<0.006){ }^{3}$ Moreover, the findings of the Age-Related Eye Disease Study Report No 5 showed a lower incidence of nuclear cataracts in diabetic patients (odds ratio, 0.68; $95 \%$ confidence interval, $0.47-0.98) .{ }^{15} \mathrm{NS}$ was originally thought to be less likely to progress in diabetic patients. Likewise, the findings of the present study showed no progression of NS in the DR-group eyes, even in the nonoperated eyes.

It should be noted that the underlying mechanism for less progression of NS in the DR-group eyes in the present study remains unclear. Previous studies have examined the distribution of oxygen in the intraocular fluids, the vitreous body, and the aqueous humor. ${ }^{16,17}$ Studies of retinal oxygenation revealed that much of the oxygen in the vitreous body is derived by diffusion from the retina. ${ }^{18-20} \mathrm{DR}$ is reportedly associated with a decreased oxygen supply to the inner retina. ${ }^{21,22}$ Since the retina is the source of most of the oxygen in the vitreous body, it seems likely that diabetic patients would have decreased levels of oxygen in the vitreous body. Increased intraocular oxygen concentration is reportedly a risk factor for the progression of $\mathrm{NSS}^{23,24}$ and a previous study reported the significantly lower intravitreal oxygen tension in diabetic patients than in nondiabetic patients $(P<0.001)$ as a reason for less progression of NS in diabetic patients. ${ }^{25}$ Lower oxygen tension results in reduced production of activated oxygen in nuclear proteins, which may inhibit the progression of lens NS in diabetic patients. ${ }^{26}$

Another possibility is that hyperglycemia may play a protective role against the oxidative damage. A previous study reported that high glucose induces an increase in antioxidant enzyme levels in human endothelial cells; ${ }^{27}$ however, it was speculated in that study that hyperglycemia may cause increased production of free radicals, and evidence supports a prominent role for these reactive molecules as mediators of endothelial cell dysfunction in diabetes. Those findings appear to be contradictory, so further investigation is necessary to fully elucidate the mechanism for less progression of NS in diabetic patients.

The vision loss attributable to NS may easily be overestimated in patients with diabetes after undergoing vitrectomy. With vitrectomy of a phakic eye in patients 50 years of age or older in Japan, lensectomy also tends to be performed. Reportedly, lensectomy, particularly in diabetic patients, may also increase the incidence of postoperative neovascular glaucoma. ${ }^{28,29}$ The findings of this present study suggest the need to reconsider the indications for simultaneous cataract surgery with vitrectomy.

\section{Acknowledgment}

The authors wish to thank John Bush for reviewing the manuscript.

\section{Disclosure}

The authors report no conflicts of interest in this work.

\section{References}

1. Cherfan GM, Michels RG, de Bustros S, Enger C, Glaser BM. Nuclear sclerotic cataract after vitrectomy for idiopathic epiretinal membranes causing macular pucker. Am J Ophthalmol. 1991;111(4):434-438.

2. Ogura Y, Takanashi T, Ishigooka H, Ogino N. Quantitative analysis of lens changes after vitrectomy by fluorophotometry. Am J Ophthalmol. 1991;111(2):179-183.

3. Michels RG. Vitrectomy for macular pucker. Ophthalmology. 1984; 91(11):1384-1388.

4. Leonard RE 2nd, Smiddy WE, Flynn HW Jr, Feuer W. Long-term visual outcomes in patients with successful macular hole surgery. Ophthalmology. 1997;104(10):1648-1652.

5. Thompson JT, Glaser BM, Sjaarda RN, Murphy RP. Progression of nuclear sclerosis and long-term visual results of vitrectomy with transforming growth factor beta-2 for macular holes. Am J Ophthalmol. 1995;119(1):48-54.

6. Smiddy WE, Feuer W. Incidence of cataract extraction after diabetic vitrectomy. Retina. 2004;24(4):574-581.

7. Samarawickrama C, Wang JJ, Burlutsky G, Tan AG, Mitchell P. Nuclear cataract and myopic shift in refraction. Am J Ophthalmol. 2007; 144(3):457-459.

8. Yata K, Fujiwara T, Yamamoto A, Ito K, Tsuyama Y. Diabetes as risk factor of cataract: differentiation by retroillumination photography and image analysis. Ophthalmic Res. 1990;22(Suppl 1):78-80.

9. West SK, Valmadrid CT. Epidemiology of risk factor for age-related cataract. Surv Ophthalmol. 1995;39(4):323-334.

10. Thompson JT. The role of patient age and intraocular gases in cataract progression following vitrectomy for macular holes and epiretinal membranes. Trans Am Ophthalmol Soc. 2003;101:485-498.

11. Li Y, Yang CX, Qing GP, Wei WB. Changes in anterior chamber depth following vitrectomy. Chin Med J (Engl). 2013;126(19): 3701-3704. 
12. Novak MA, Rice TA, Michels RG, Auer C. The crystalline lens after vitrectomy for diabetic retinopathy. Ophthalmology. 1984;91(12): $1480-1484$.

13. de Bustros S, Thompson JT, Michels RG, Rice TA. Vitrectomy for progressive proliferative diabetic retinopathy. Arch Ophthalmol. 1987; 105(2):196-199.

14. Schachat AP, Oyakawa RT, Michels RG, Rice TA. Complications of vitreous surgery for diabetic retinopathy. II. Postoperative complications. Ophthalmology. 1983;90(5):522-530.

15. Age-Related Eye Disease Study Research Group. Risk factors associated with age-related nuclear and cortical cataract: a case-control study in the Age-Related Eye Disease Study, AREDS Report No 5. Ophthalmology. 2001;108(8):1400-1408.

16. Maeda N, Tano Y. Intraocular oxygen tension in eyes with proliferative diabetic retinopathy with and without vitreous. Graefes Arch Clin Exp Ophthalmol. 1996;234(Suppl 1):S66-S69.

17. Kwan M, Niinikoski J, Hunt TK. In vivo measurements of oxygen tension in the cornea, aqueous humor, and anterior lens of the open eye. Invest Ophthalmol. 1972;11(2):108-114.

18. Alder VA, Yu DY, Cringle SJ. Vitreal oxygen tension measurements in the rat eye. Exp Eye Res. 1991;52(3):293-299.

19. Ito Y, Berkowitz BA. MR studies of retinal oxygenation. Vision Res. 2001;41(10-11):1307-1311.

20. Yu DY, Cringle SJ, Alder VA. The response of rat vitreal oxygen tension to stepwise increases in inspired percentage oxygen. Invest Ophthalmol Vis Sci. 1990;31(12):2493-2499.

21. Riva CE, Logean E, Falsini B. Visually evoked hemodynamical response and assessment of neurovascular coupling in the optic nerve and retina. Prog Retin Eye Res. 2005;24(2):183-215.
22. Shonat RD. Oxygen delivery to the retina and related visual pathology. Brief review. Adv Exp Med Biol. 2003;510:249-254.

23. Barbazetto IA, Liang J, Chang S, Zheng L, Spector A, Dillon JP. Oxygen tension in the rabbit lens and vitreous before and after vitrectomy. Exp Eye Res. 2004;78(5):917-924.

24. Holekamp NM, Shui YB, Beebe DC. Vitrectomy surgery increases oxygen exposure to the lens: a possible mechanism for nuclear cataract formation. Am J Ophthalmol. 2005;139(2):302-310.

25. Holekamp NM, Shui YB, Beebe D. Lower intraocular oxygen tension in diabetic patients: possible contribution to decreased incidence of nuclear sclerotic cataract. Am J Ophthalmol. 2006;141(6):1027-1032.

26. Holekamp NM, Bai F, Shui YB, Almony A, Beebe DC. Ischemic diabetic retinopathy may protect against nuclear sclerotic cataract. Am J Ophthalmol. 2010;150(4):543-550.e1.

27. Ceriello A, dello Russo P, Amstad P, Cerutti P. High glucose induces antioxidant enzymes in human endothelial cells in culture. Evidence linking hyperglycemia and oxidative stress. Diabetes. 1996;45(4):471-477.

28. Blankenship GW. The lens influence on diabetic vitrectomy results. Report of a prospective randomized study. Arch Ophthalmol. 1980;98(12):2196-2198.

29. Rice TA, Michels RG, Maguire MG, Rice EF. The effect of lensectomy on the incidence of iris neovascularization and neovascular glaucoma after vitrectomy for diabetic retinopathy. Am J Ophthalmol. 1983;95(1):1-11.
Clinical Ophthalmology

\section{Publish your work in this journal}

Clinical Ophthalmology is an international, peer-reviewed journal covering all subspecialties within ophthalmology. Key topics include: Optometry; Visual science; Pharmacology and drug therapy in eye diseases; Basic Sciences; Primary and Secondary eye care; Patient Safety and Quality of Care Improvements. This journal is indexed on

Submit your manuscript here: http://www.dovepress.com/clinical-ophthalmology-journal

\section{Dovepress}

PubMed Central and CAS, and is the official journal of The Society of Clinical Ophthalmology (SCO). The manuscript management system is completely online and includes a very quick and fair peer-review system, which is all easy to use. Visit http://www.dovepress.com/ testimonials.php to read real quotes from published authors. 